\title{
In vivo Modular Control Analysis of Energy Metabolism in Contracting Skeletal Muscle
}

\section{Laurent M. Arsac, Christophe Beuste, Sylvain Miraux, Véronique Deschodt-Arsac, Eric Thiaudière, Jean-Michel FRANCONI, Philippe H. Diolez}

Résonance Magnétique des Systèmes Biologiques, UMR 5536 Centre National de la Recherche Scientifique (CNRS) - Université Bordeaux 2, Bordeaux, France.

SHORT TITLE : in vivo modular control analysis of muscle energetics 


\section{SYNOPSIS}

We used ${ }^{31} \mathrm{P}$ MR spectroscopy measurements of energetic intermediates (ATP, Pi, PCr) in combination with the analytical tools of metabolic control analysis to study in vivo energy metabolism in contracting skeletal muscle of anesthetized rats over a broad range of workload. According to our recent Modular Control Analysis (MoCA) used to describe regulatory mechanisms in beating heart, we defined the energetic system of muscle contraction as two modules (PCr-Producer and PCr-Consumer) connected by the energetic intermediates.

Hypoxia and electrical stimulation were used in this in vivo study as reasonably selective modulations of Producer and Consumer respectively. As quantified by elasticity coefficients, the sensitivities of each module to PCr determine the control of steady state contractile activity and metabolite concentrations. The magnitude of the elasticity of the producer was high (4.3 \pm 0.6$)$ at low workloads and decreased 5-fold (to $0.9 \pm 0.2$ ) at high workloads. By contrast, the elasticity of the consumer remained low $(0.5$ to 1.2$)$ over the range of metabolic rates studied. The control exerted by each module over contraction was calculated from these elasticities. The control of contraction was found on the consumer at low workloads then swung to the producer, due to the workload-dependent decrease in the elasticity of producer.

The workload-dependent elasticity and control pattern of energy production in muscle is a major difference with heart. Since module rate and elasticity depend on the concentrations of substrates and products, the absence of homeostasis of the energetic intermediates in muscle, by opposition to heart, may likely be the origin of the workload-dependent elasticity of the producer module.

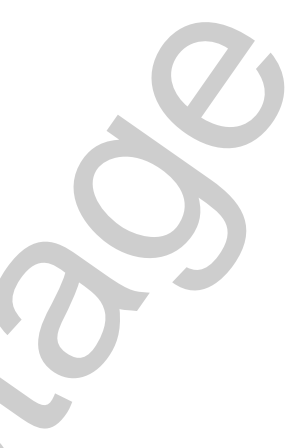

Muscle energetics ; elasticity coefficient ; control coefficient ; metabolic regulation ; ${ }^{31}$ P MR spectroscopy ; Modular Control Analysis 


\section{INTRODUCTION}

In skeletal muscle as in heart, contraction is powered up by the Gibbs free energy of ATP hydrolysis $(\Delta \mathrm{Gp})$ available at actomyosin ATPases level. In the absence of changes in regulatory external effectors, both rates of ATP supply and ATP demand depend on the concentration of their substrates/products ATP, ADP and Pi. The concentration of ATP remains fairly constant during muscle contraction and [ADP] remains low and undetectable by non-invasive techniques like ${ }^{31} \mathrm{P}$ magnetic resonance spectroscopy. As far as the cytosolic creatine kinase reaction remains close to equilibrium, [PCr] reflects the status of energy balance within the entire myocyte and over all the fibres in the studied muscles [1,2]. Since energy production and energy consumption during muscle contraction are respectively responsible for increasing and decreasing PCr concentration, ${ }^{31} \mathrm{P}$ MRS assessments of PCr levels are representative of cellular energetic intermediates, and have been widely used to study the behaviour of the energetic system of contraction in heart as well as in muscle [3-9].

Recently, our group proposed a new experimental approach to describe control and regulatory mechanisms of energetics in intact beating hearts, based on the development of the Modular Control Analysis (MoCA). Heart energetics was defined as a two-module system. Modules respectively produce (so-called Producer) and consume (Consumer) energy and are connected by PCr concentration [10]. Central to this approach are the elasticity coefficients of the ATP/PCr producing and ATP/PCr consuming modules to PCr concentration [10]. Indeed, elasticity values in such a modular system quantify how the system is maintained in a steady state. Elasticities also allow to quantify how the effects of an external effector signal/molecule that change the steady state are transmitted through the intermediate [10-13].

It is worth noting that heart is capable of dramatically altering its overall energy flux with only minimal changes in the concentrations of energetic intermediates [14]. Homeostasis of the intermediates in heart is likely the consequence of a perfectly balanced parallel activation of ATP supply and ATP demand [14, 15] as demonstrated in intact hearts stimulated by either calcium [10] or adrenaline [16]. By comparison, an unbalanced activation could thus be suspected in skeletal muscle $[5,15,17]$ since the transition from low to high work intensity is consistently accompanied by a decrease level of $\mathrm{PCr}$ and an increase in the connected intermediates (free creatine, ADP and Pi) [3, 4, 7, 9, 18, 19]. These changes in the energetic intermediates may in turn impact the kinetics of the modules. Therefore, it appears that the in vivo determination of the elasticities of $\mathrm{ATP} / \mathrm{PCr}$ producing and consuming modules over a wide range of activity is of great interest in skeletal muscle. 
For this purpose, we developed a non-invasive experimental setup providing simultaneous in vivo assessments of $\mathrm{PCr}$ content and contraction force from electrically stimulated rat muscle. Plantar flexor muscles of intact anesthetized rats were interrogated by a two-probe $\left({ }^{1} \mathrm{H}\right.$ and $\left.{ }^{31} \mathrm{P}\right)$ setup providing muscle imaging at rest, and muscle content of phosphorylated intermediates during sustained contractions. The experimental design was defined in the frame of MoCA: the Consumer module contains all the reactions that consume ATP/PCr in contracting myocytes, and the Producer module grouped the reactions catalysed by mitochondria and glycolysis together with substrates delivery to contracting muscles. Under steady state conditions, the selective modulation of $\mathrm{ATP} / \mathrm{PCr}$ production required by MoCA was achieved by changing the oxygen concentration in the gas mixture inhaled by the rat. The other required modulation - ATP/PCr consumption - was carried out by decreasing the electrical stimulation of plantar flexors. The energetic flux through this two-module system is represented by the ATP turnover rate. In contracting muscles during sustained submaximal exercise, the ATP turnover rate is a direct function of the work rate by contraction [20]; therefore the later can be used as an index of the former [21].

We carried out in vivo the analytical study by MoCA of energy metabolism in contracting muscle over a broad range of metabolic rates. The full MoCA analysis provided a complete integrative description of the behaviour of muscle energetics for each individual animal tested. In agreement with previously modelled elasticities [5], we demonstrate here that the sensitivity of (mitochondrial) ATP/PCr production in vivo decreases as a function of activity in skeletal muscle. 


\section{METHODS}

\section{Animals}

Female Wistar rats weighing 280-340g were used according to the guidelines of the National Research Council Guide for the care and use of laboratory animals. Rats were housed in an environmentally controlled facility (12-h light 12 -h dark cycle, $\left.22^{\circ} \mathrm{C}\right)$, and received water and food ad libitum.

Rats were anesthetized by continuous inhalation of a gas mixture containing $1.5 \%$ isoflurane delivered in a face-mask during a 90-min period corresponding to the duration of the experiment into the magnet, then housed back in their environmentally controlled facility.

\section{Muscle stimulation}

Plantar flexor muscles of rats in supine position were stimulated $(3 \mathrm{~Hz}$, pulse duration $220 \mu \mathrm{s})$ directly with transcutaneous surface electrodes located at the knee and heel levels [18] and connected to a Compex 2 stimulator (Compex Médical SA, Ecublens, Switzerland). Current intensity was set to $4 \mathrm{~mA}$ (low workload), 5-6mA (moderate workload) and 7-8mA (high workload). MRS acquisition was synchronized to electrical stimulation. Electrically stimulated regions of the plantar flexor group were assessed by $\mathrm{T}_{2}$-mapping MRI [18]. Transverse relaxation times constants $\left(\mathrm{T}_{2}\right)$ were measured using an imaging CPMG (CarrPurcell-Meiboom-Gill) pulse sequence (64 echoes, echo time: $7.3 \mathrm{~ms}$, repetition time : $2000 \mathrm{~ms}$ ). $\mathrm{T}_{2}$-maps indicated that the mixed-gastrocnemius muscle responded to electrical stimulation over the range of stimulations used (figure 2).

\section{${ }^{31} P$ magnetic resonance spectroscopy}

Rats were placed in a supine position into a supraconducting magnet (4.7 Tesla, 47/50 Biospec Avance MR system (Brüker, Karlsruhe, Germany). The foot was positioned on the ergometer pedal and the hindlimb was immobilized so that the lower limb was centred inside a $30 \mathrm{~mm}$ diameter ${ }^{1} \mathrm{H}$ Helmoltz imaging coil tuned to the $1 \mathrm{H}$ frequency (200.3 MHz).

A circular homebuilt transmit-receive probe $(18 \mathrm{~mm})$ was placed horizontally under the calf and was tuned to the $31 \mathrm{P}$ frequency $(81.1 \mathrm{MHz})$. Field homogeneity was achieved locally in a $20 \times 20 \times 20 \mathrm{~mm}^{3}$ voxel by using the water proton resonance, with typical line widths of $30-35 \mathrm{~Hz}$.

${ }^{31} \mathrm{P}$ free-induction decays (FIDs) $\left(100 \mu \mathrm{s}\right.$ rectangular pulse, $60^{\circ}$ flip angle at the centre of the coil; 40 accumulations; $2.8 \mathrm{~s}$ recovery time; $3.3 \mathrm{kHz}$ spectral width; 1024 data points) were acquired in $111 \mathrm{~s}$ blocks throughout the experimental protocol. 
After Fourier transform, the NMR spectra were then routinely deconvolved into Lorentzian lines (Igor Pro, Wavemetrics, Oregon USA). The phosphocreatine, Pi, and ß-ATP peak areas were calculated and were converted to concentrations using $[\mathrm{ATP}]=8.2 \mathrm{mM}$. Intracellular $\mathrm{pH}$ was determined by using the chemical shift of Pi relatively to phosphocreatine.

\section{Contraction measurements}

Contraction resulting from muscle electrical stimulation was measured with a homebuilt ergometer consisting in a foot pedal connected to a hydraulic piston. A hydraulic circuit filled with water connected the piston to a force transducer (MLT0699 + Powerlab, AD Instruments, Bella Vista, Australia), which was placed outside the magnet. The pedal was adjusted so that the foot was perpendicular to the leg. Changes in pressure induced by pedal stroke were recorded every $5 \mathrm{~ms}$. The magnitude of strokes was computed as a function of time (Igor, Wavemetrics, Oregon, USA) to provide the contraction signal. Since only relative changes (in \%) in contraction are required for MoCA, contraction force was expressed as the voltage delivered by the sensor ( $\mu$ volts, see figure 3 ).

\section{The modular system}

We defined the system of contraction as two modules, Producer and Consumer connected by the phosphorylation potential $(\Delta \mathrm{Gp})$ as represented by $[\mathrm{PCr}]$ in our conditions $[10,16,22]$. By grouping reactions and reactants into large modules connected by a small number of explicit intermediates, complex systems are thus simplified [13, 23]. The behaviour of the system was studied over a wide range of steady states achieved by sustained steady level of electrical stimulation. The elasticity coefficients of Producer and Consumer were determined under each given steady state conditions. The typical design of each experiment is described in figure 1. Two ${ }^{31} \mathrm{P}-\mathrm{MR}$ spectra (spectra 1 and 2 ) were acquired before muscle stimulation began to assess phosphocreatine concentration at rest ([PCr $\left.]_{\text {rest }}\right)$. During spectra 3 to 12 , muscle stimulation was progressively increased up to the desired current intensity, and hypoxia was gradually initiated by reduction of the $\mathrm{FiO}_{2}$ of breathed gas to $9 \% \mathrm{O}_{2}$ (in $\mathrm{N}_{2}$ ). The system reached thus a hypoxic steady state where the producer is inhibited for several minutes by hypoxia (spectra 14 to 18). During spectrum 19, hypoxia was stopped; the rat then inspired air and the system achieved quickly the so-called 'reference' steady state (absence of modulation, spectra 20 to 24). The consumer elasticity was calculated from relative changes in steady levels of PCr and contraction between 'reference' and hypoxic conditions (figure 3). During the last steady state period, the electrical stimulation was decreased by $1 \mathrm{~mA}$ to lessen energy consumption while $\mathrm{F}_{\mathrm{i}} \mathrm{O}_{2}$ was purposely maintained at 0.21 (ambient air). The steady 
values during this stage when compared to 'reference' were used for the calculation of the producer elasticity (figure 3). The elasticity coefficients in each individual animal were quantified by dividing relative change in contraction by relative changes in [ $\mathrm{PCr}]$ [10]. The single-experiment determination of the elasticities developed here allows the determination of the control distribution for each animal studied. The contribution of each module to the control over the flux is quantified by a parameter called the flux control coefficient, defined as the ratio of the relative change in a given flux caused by a small relative change in the module activity. While the elasticity is a property of the module, the control coefficient is a property of the whole system. Control coefficients over the contraction energy flux $\left(C_{p}\right.$ and $\left.C c\right)$ were calculated from the experimental measurements of elasticities [10].

\section{Statistics}

Data are presented as mean \pm SD. Elasticity and control coefficients at low, moderate and high work rates were compared using one-way analysis of variance. Significant differences were considered at $P<0.05$.

\section{RESULTS AND DISCUSSION}

In the present study, we experimentally apply for the first time metabolic control analysis in vivo to study non-invasively the behaviour of energy metabolism in contracting skeletal muscle on an anesthetized rat. The analysis was carried out using controlled electrical stimulation to provide a wide range of steady muscle activity. Our experimental two-probe setup $\left({ }^{1} \mathrm{H}\right.$ and $\left.{ }^{31} \mathrm{P}\right)$ also allowed the application of a specific MRI technique $\left(\mathrm{T}_{2}\right.$-mapping) dedicated to identify the contracting regions inside the studied muscle group [18]. Figure 2 shows typical axial $\mathrm{T}_{2}$-maps from the rat lower hindlimb obtained at rest (a) and immediately following low (b) and high (c) steady stimulated contraction. Although this technique did not indicate which precise fibres were contracting, the images in Figure 2 clearly show that only gastrocnemius mixed-muscles responded to the electrical stimulation in the current experimental setup.

Determination of elasticity coefficients. In the formalism of MoCA and more extensively in metabolic control analysis, the correct determination of elasticities must obey some rules: only the intermediate and the flux must vary freely while other parameters like external effector molecules or signals must remain unchanged. The present non-invasive MRS experiments in vivo could therefore be considered as a preliminary crude method since 
modulations of the steady state by hypoxia and electrical stimulation might not be perfectly specific. Hypoxia evidently affects the PCr producing module via selective mitochondrial inhibition, resulting in both [PCr] decrease and consequent contraction decrease (table 1 and figure 3). However, the modulation of the consumer module by changing electrical stimulation may be less selective: some cytosolic factors, e.g. intracellular calcium pulses, might impact the producer module in some extent. It is yet of note that in contrast with cardiac muscle wherein direct activation of Producer by calcium has been demonstrated, $\left[\mathrm{Ca}^{2+}\right]$ changes may likely be of minor concern in the interpretation of skeletal muscle results. This is illustrated by the dynamics of [PCr] and oxygen consumption in perfused cat soleus (oxidative muscle) as compared to biceps brachii (dominantly glycolytic muscle) [2]: a postexercise overshoot of [PCr] was detectable only in soleus but not in biceps brachii, indicating the persistence of the activation of PCr production only in the electrically stimulated fully oxidative muscle. $\mathrm{T}_{2}$-maps indicate that only gastrocnemius muscle groups are stimulated in the present study. Since the rat gastrocnemius is not a fully aerobic muscle [24], the direct activation of $\mathrm{PCr}$ production by slight changes in electrical stimulation is minimized. A strong additional argument comes from the integration of a broad range of experimental data from skeletal muscle into a dynamic computer model of oxidative phosphorylation and ATP usage $[15,17,25]$. Indeed, to fit with concerned data, the model predicts the absence of direct activation of PCr production in electrically stimulated muscle [17].

We may therefore consider that the present in vivo application of MoCA to skeletal muscle energetics might be a crude method and the experimentally determined values of the elasticity coefficient would be approximated. However, we assume that under our conditions, this would not change the general conclusions (see below) and the development of a quantitative approach in vivo, even based on a crude method seems yet to be advantageous [5, $6,26]$.

The intermediate. In the energy metabolism of contracting skeletal muscle, the true intermediate between production and consumption is the phosphorylation potential $\Delta \mathrm{Gp}$. A direct measurement of $\Delta \mathrm{Gp}$ in vivo is difficult. Although $\Delta \mathrm{Gp}$ can be calculated from MRS of phosphorylated intermediates and $\mathrm{pH}$, errors in the detection of $\mathrm{Pi}, \mathrm{PCr}$, and each ATP peak sum up in this calculation. Thus, we based our analysis on [ $\mathrm{PCr}]$ changes, taking into account the reliable signal-to-noise ratio obtained in vivo with our setup (figure 3). Furthermore, the phosphorylation potential depends on the concentrations of ATP, ADP, Pi and $\mathrm{H}^{+}$, which are all directly related to $\mathrm{PCr}$ concentration and therefore changes in $\mathrm{PCr}$ directly reflects changes in $\Delta \mathrm{Gp}$. Since only relative changes in the intermediate (and flux) are considered in the 
modular control analysis, $\mathrm{PCr}$ may be used as a representative of $\Delta \mathrm{Gp}$ in this study [10]. Under our conditions, changes in $\left[\mathrm{H}^{+}\right]$have negligible effects on $\Delta \mathrm{Gp}$ (e.g. a drop in muscle $\mathrm{pH}$ by 0.15 only corresponds to less than $2 \%$ change in $\Delta \mathrm{Gp}$ ), and the negligible effect of $\mathrm{pH}$ on myofibrils has been demonstrated in skeletal muscle at physiological temperature [27]. At last, muscle $\mathrm{pH}$ was assessed in our MRS experiment in vivo and values reported in table 1 show only very small changes in $\mathrm{pH}$ over the workload range tested. In conclusion, in vivo MRS assessments of $\mathrm{PCr}$ changes are relevant to study the kinetic response of the $\mathrm{PCr}$ Producer and PCr Consumer.

Experimental elasticities and muscle activity. Quantifying elasticity coefficients in the frame of MoCA requires the simultaneous measurements of relative changes in $[\mathrm{PCr}]$ and in contraction power output. This was achieved here in vivo as illustrated by typical ${ }^{31} \mathrm{P}-\mathrm{MR}$ spectra and contraction measurements presented in figure 3. Such a typical experiment was repeated under various levels of electrical stimulation, thus allowing the quantification of elasticity coefficients over a wide range of skeletal muscle activity. The activity has been scaled according to the steady-state level of $[\mathrm{PCr}]$ relatively to $[\mathrm{PCr}]$ at rest, since a strong correlation has been demonstrated between this parameter and the relative workload in human muscle [4]. The PCr level varied from $82 \%$ at the lowest workload to $38 \%$ [PCr]rest at the highest workload. Experimental values for $[\mathrm{PCr}]$ and contraction were arbitrary grouped as low, moderate and high level of muscle activity. Activity-specific averaged values of PCr and contraction are presented in table 1 . These values are only indicative since the present study was designed to allow single-animal comprehensive MoCA analysis, so that elasticity coefficients were actually calculated for each animal. Therefore, between-animal variability, as indicated by standard deviation in table 1 , does not interfere with the calculation of elasticities. Averaged individual elasticities measured experimentally are reported in table 2. The statistical analysis indicates that the Producer elasticity to $\mathrm{PCr}$ decreased more than 5fold from low to high workloads (from -6.7 to -0.3 ). The Consumer elasticity to PCr was much lower than that of Producer and remained roughly unchanged $( \pm 1.0)$ when the workload changed. Figure 4 shows the results from individual experiments and provides a clear picture of the activity-dependent value of the Producer elasticity to $\mathrm{PCr}$ and of the roughly constant Consumer elasticity to PCr. Taking into account these marked differences in elasticity values, and the strong effect of muscle activity on the producer elasticity, the even crude method developed here in vivo provides for the first time a clear picture of the behaviour of the energetic system in contracting skeletal muscle in the in vivo integrative context. 
The shift in kinetic control over contraction. The respective control coefficients of the producer and the consumer were calculated from the above elasticity coefficients as allowed by MoCA [10]. Therefore we can describe how control over contraction is distributed in our conditions. The major finding here is that the control distribution is not unique but depends on muscle activity, in agreement with previous predictions [5]. The change in the control distribution was almost completely accounted for by the decrease in the producer elasticity. At low activity, the producer had a very high elasticity and consequently very low control $(10 \%)$ over contraction (table 2 and fig $4 \mathrm{~b}$ ). Under these conditions, energy consumption mainly set by the activity of the AM-ATPases and SR-ATPases controls the contraction (90\%), i.e. ATP turnover. At moderate activity, due to the elasticity decrease, the control of the producer module over the contraction increased but remained low $(25 \%)$; whereas the control by consumer module shifted down to $75 \%$. Under high activity conditions, where $\mathrm{PCr}$ depletion was above $50 \%$, the control distribution changed dramatically: the producer rather than the consumer had dominant control over contraction. It was even observed in some animals that the energy production controlled almost totally ( $>80 \%$ ) contraction (fig $4 \mathrm{~b}$ ). Again, it is unlikely that approximations in the experimental determination of elasticity due to our in vivo method can modify the control pattern in a large extent.

\section{CONCLUSION}

For the first time, the behaviour of the energy metabolism in contracting skeletal muscles under steady state conditions has been quantified here experimentally in vivo in anesthetized rats. Despite the difficulty to apply strictly selective modulations of energy production and consumption in in vivo skeletal muscle, the values of the elasticity coefficients indicate clearly how the steady state of contraction is maintained in skeletal muscle over a wide range of metabolic rates. We highlighted here the low sensitivity of the energy-consuming ATPases to reactants at every metabolic rate as compared to energy production and concluded therefore that, over the range of activity studied here, the activation of myofibrils is strongly dependent on the direct stimulation by calcium. By contrast, the oxidative phosphorylation processes included in the Producer module appeared highly reactive to small changes in the intermediates at low skeletal muscle activity, so that feedback metabolic activation is one possible - although not exclusive - mechanism of activation for energy production. This mechanism is far less efficient in activating energy production at higher level of muscle 
activity, as indicated by the activity-dependent decrease in the producer elasticity, not observed in the heart $[10,14,28]$.

It sounds crucial to understand the mechanisms underlying the observed changes in the Producer elasticity with activity in skeletal muscle but not in heart. In heart, due to the perfectly balanced parallel activation of energy supply and energy demand by external effectors $[10,14,16], \mathrm{PCr}$ (as well as the other energetic metabolites and $\Delta \mathrm{Gp}$ ) does not change as a function of contractile activity.

As concentrations of all reactants remain constant, the local kinetic response of each connected module is constant as well. By contrast, the drop in $[\mathrm{PCr}]$ in skeletal muscle when activity increases illustrates substantial changes in all reactants likely due to unbalanced activation. We hypothesize here that the producer elasticity dependence on activity could be due to these changes in reactant concentrations highlighted by MRS assessments of PCr levels (table 1). By contrast with the absence of modification of consumer response to the intermediates, results indicates that producer module kinetics shows substrate saturation (probably by creatine) so that its response to substrate changes decreases at high workload and the elasticity drops to the low values observed.

As we still did not test the regulatory effect of any effector on the system - the effects responsible for a change in the steady state in the formalism of MoCA - no conclusion can be made about the regulation of muscle energetics in vivo. The magnitude of respective activation of energy supply and energy demand when muscle activity increases [16] cannot be detected by our measurements of elasticity and flux control coefficients. To date only approaches in silico have provided insights about intact skeletal muscle [15, 17, 25, 29, 30] while experimental evidences were obtained recently in intact heart $[10,16]$.

We anticipate that the application of MoCA analysis, will be of great interest for integrative biology studies of healthy or pathological muscles, since it allows deciphering with the biochemistry of energy transfer into the physiology of muscle cells. At this stage, the application of MoCA to skeletal muscle energetics in vivo may be considered as a semiquantitative approach, because of methological limitations, but could well be an advance in relation to purely intuitive considerations. Other semi-quantitative approaches have been demonstrated very helpful in this area $[6,29]$. MoCA has the potential to highlight defects/adaptations in the energy transfer system on the basis of elasticity changes. It is unknown to date whether physical activity, chronic hypoxia or pathologies have the potential to alter elasticities or whether only modules activities are affected. The design and setup of 
the present experimental approach provide a useful tool to investigate non-invasively the primary target(s) responsible for these various alterations of muscle contraction.

\section{Acknowledgments}

This work was supported by: Centre National de la Recherche Scientifique, Université Victor Segalen Bordeaux 2, Conseil Régional de la Région Aquitaine and Institut Fédératif de Recherche 4, Bordeaux, France. 


\section{REFERENCES}

1 Kushmerick, M. J. (1998) Energy balance in muscle activity: simulations of ATPase coupled to oxidative phosphorylation and to creatine kinase. Comp Biochem Physiol B Biochem Mol Biol 120, 109-123

2 Kushmerick, M. J., Meyer, R. A. and Brown, T. R. (1992) Regulation of oxygen consumption in fast- and slow-twitch muscle. Am J Physiol 263, C598-606

3 Hochachka, P. W. and McClelland, G. B. (1997) Cellular metabolic homeostasis during large-scale change in ATP turnover rates in muscles. J Exp Biol 200, 381-386

4 Hogan, M. C., Richardson, R. S. and Haseler, L. J. (1999) Human muscle performance and PCr hydrolysis with varied inspired oxygen fractions: a 31P-MRS study. J Appl Physiol 86, 1367-1373

5 Jeneson, J. A. (2002) Regulation analysis of contractile ATPase flux in skeletal muscle. Mol Biol Rep 29, 167-170

6 Jeneson, J. A., Westerhoff, H. V. and Kushmerick, M. J. (2000) A metabolic control analysis of kinetic controls in ATP free energy metabolism in contracting skeletal muscle. Am J Physiol Cell Physiol 279, C813-832

7 Jones, A. M., Wilkerson, D. P and Fulford, J. (2008) Muscle [phosphocreatine] dynamics following the onset of exercise in humans: the influence of baseline workrate. J Physiol 586, 889-898

8 Matheson, G. O., Allen, P. S., Ellinger, D. C., Hanstock, C. C., Gheorghiu, D., McKenzie, D. C., Stanley, C., Parkhouse, W. S. and Hochachka, P. W. (1991) Skeletal muscle metabolism and work capacity: a 31P-NMR study of Andean natives and lowlanders. J Appl Physiol 70, 1963-1976

9 Paganini, A. T., Foley, J. M. and Meyer, R. A. (1997) Linear dependence of muscle phosphocreatine kinetics on oxidative capacity. Am J Physiol 272, C501-510

10 Diolez, P., Deschodt-Arsac, V., Raffard, G., Simon, C., Santos, P. D., Thiaudiere, E., Arsac, L. and Franconi, J. M. (2007) Modular regulation analysis of heart contraction: application to in situ demonstration of a direct mitochondrial activation by calcium in beating heart. Am J Physiol Regul Integr Comp Physiol 293, R13-19

11 Ainscow, E. K. and Brand, M. D. (1999) Quantifying elasticity analysis: how external effectors cause changes to metabolic systems. Biosystems 49, 151-159

12 Brand, M. D. (1997) Regulation analysis of energy metabolism. J Exp Biol 200, 193202

13 Brand, M. D. and Curtis, R. K. (2002) Simplifying metabolic complexity. Biochem Soc Trans 30, 25-30 
14 Balaban, R. S. (2002) Cardiac energy metabolism homeostasis: role of cytosolic calcium. J Mol Cell Cardiol 34, 1259-1271

15 Korzeniewski, B. (2007) Regulation of oxidative phosphorylation through parallel activation. Biophys Chem 129, 93-110

16 Korzeniewski, B., Deschodt-Arsac, V., Calmettes, G., Franconi, J. M. and Diolez, P. H. (2008) Physiological heart activation by adrenaline involves parallel activation of ATP usage and supply. Biochem J

17 Korzeniewski, B. (2003) Regulation of oxidative phosphorylation in different muscles and various experimental conditions. Biochem J 375, 799-804

18 Giannesini, B., Izquierdo, M., Le Fur, Y., Cozzone, P. J., Fingerle, J., Himber, J., Kunnecke, B., Von Kienlin, M. and Bendahan, D. (2005) New experimental setup for studying strictly noninvasively skeletal muscle function in rat using $1 \mathrm{H}$-magnetic resonance (MR) imaging and 31P-MR spectroscopy. Magn Reson Med 54, 1058-1064

19 Haseler, L. J., Richardson, R. S., Videen, J. S. and Hogan, M. C. (1998) Phosphocreatine hydrolysis during submaximal exercise: the effect of FIO2. J Appl Physiol 85, 1457-1463

20 Arthur, P. G., Hogan, M. C., Bebout, D. E., Wagner, P. D. and Hochachka, P. W. (1992) Modeling the effects of hypoxia on ATP turnover in exercising muscle. J Appl Physiol 73, 737-742

21 Nioka, S., Argov, Z., Dobson, G. P., Forster, R. E., Subramanian, H. V., Veech, R. L. and Chance, B. (1992) Substrate regulation of mitochondrial oxidative phosphorylation in hypercapnic rabbit muscle. J Appl Physiol 72, 521-528

22 Diolez, P., Raffard, G., Simon, C., Leducq, N., Dos, S. P. and Canioni, P. (2002) Mitochondria do not control heart bioenergetics. Mol Biol Rep 29, 193-196

23 Brown, G. C., Hafner, R. P. and Brand, M. D. (1990) A 'top-down' approach to the determination of control coefficients in metabolic control theory. Eur J Biochem 188, 321-325

24 Bahi, L., Koulmann, N., Sanchez, H., Momken, I., Veksler, V., Bigard, A. X. and Ventura-Clapier, R. (2004) Does ACE inhibition enhance endurance performance and muscle energy metabolism in rats? J Appl Physiol 96, 59-64

25 Korzeniewski, B. (1998) Regulation of ATP supply during muscle contraction: theoretical studies. Biochem J 330 ( Pt 3), 1189-1195

Wu, F., Jeneson, J. A. and Beard, D. A. (2007) Oxidative ATP synthesis in skeletal muscle is controlled by substrate feedback. Am J Physiol Cell Physiol 292, C115-124 
27 Westerblad, H., Bruton, J. D. and Lannergren, J. (1997) The effect of intracellular pH on contractile function of intact, single fibres of mouse muscle declines with increasing temperature. J Physiol 500 ( Pt 1), 193-204

28 Balaban, R. S. (1990) Regulation of oxidative phosphorylation in the mammalian cell. Am J Physiol 258, C377-389

29 Korzeniewski, B., Harper, M. E. and Brand, M. D. (1995) Proportional activation coefficients during stimulation of oxidative phosphorylation by lactate and pyruvate or by vasopressin. Biochim Biophys Acta 1229, 315-322

30 Liguzinski, P. and Korzeniewski, B. (2006) Metabolic control over the oxygen consumption flux in intact skeletal muscle: in silico studies. Am J Physiol Cell Physiol 291, C1213-1224 
Workload

\begin{tabular}{ccc}
\hline $\begin{array}{c}\text { Low } \\
(n=7)\end{array}$ & $\begin{array}{c}\text { Moderate } \\
(n=17)\end{array}$ & $\begin{array}{c}\text { High } \\
(n=9)\end{array}$ \\
$77 \pm 3 \%$ & $60 \pm 4 \%$ & $44 \pm 4 \%$ \\
$7.01 \pm 0.04$ & $6.98 \pm 0.03$ & $6.96 \pm 0.04$
\end{tabular}

Modulations around the Reference steady state

PCr-low supply

PCr-Reference

PCr-low demand

Contraction-low supply

Contraction-Reference

Contraction-low demand
$16.7 \pm 2.1 * \dagger$

$20.2 \pm 1.8$

$23.5 \pm 2.5 \div$

$279 \pm 197$

$318 \pm 172$

$168 \pm 87$
$10.7 \pm 2.4 * \dagger$

$16.3 \pm 1.4$

$12.4 \pm 1.0$

$19.1 \pm 2.7 \ddagger$

$14.4 \pm 1.3$

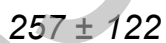

$273 \pm 145$

$323 \pm 142$

$355 \pm 162$

$261 \pm 138$ of variance and post-hoc Tukey HSD tests. ${ }^{*} P<0.05$, between low supply and reference; $\dagger P<0.05$, between low supply and low demand; $\ddagger P<0.05$, between low demand and reference. 
Workload

\begin{tabular}{ccc}
\hline Low & Moderate & High \\
$(n=7)$ & $(n=17)$ & $(n=9)$
\end{tabular}

Producer elasticity

Consumer elasticity

Control by Producer

Control by Consumer

$$
-4.3 \pm 1.7 * \dagger
$$

$0.6 \pm 0.3 \dagger$

$11 \pm 3 \% \dagger$

$89 \pm 3 \% \dagger$
$-1.6 \pm 0.9$

$0.5 \pm 0.3$

$25 \pm 9 \%$

$75 \pm 9 \%$
$-0.9 \pm 0.6$

$1.2 \pm 0.4 \ddagger$

$$
59 \pm 16 \% \ddagger
$$

$41 \pm 16 \%+$

Table 2. Elasticity and control coefficients as a function of muscle workload. Data shows the average values of the individual coefficients measured during each experiment. Values are \pm SD The effect of worload was tested by one-way analysis of variance and posthoc Tukey HSD tests. ${ }^{*} P<0.001$ between Low and Moderate, $\uparrow P<0.001$ between Low and High, $\$ P<0.001$ between High and Moderate (except consumer elasticity $P<0.05$ ). 
Figure 1: Experimental protocol. Two ${ }^{31} \mathrm{P}$ spectra were obtained from plantar flexor muscles at rest and then muscles were electrically stimulated for about one hour. After a 20-min period, modulations around the "reference" steady state were achieved, beginning with the steady state under hypoxic conditions (low supply, $9 \% \mathrm{O}_{2}$ ); then, after the switch to normal air, the reference conditions were maintained for 12 minutes. To achieve the "low demand" episode, the consumer was modulated by a slight decrease in electrical stimulation. Simultaneous measurements of ${ }^{31} \mathrm{P}$ spectra and muscle contraction during modulation of the steady state allowed calculation of the elasticity coefficient of each module. The value for $n$ depends on the desired imposed workload (see Methods section).

Figure $2:{ }^{1}$ H-MRI study of the lower hindlimb of the anesthetized rat placed in the experimental setup. (a) typical $\mathrm{T}_{2}$-weighted image obtained from the axial view at rest. (b) $\mathrm{T}_{2}$-maps obtained immediately after a typical steady state under low electrical stimulation and (c) high electrical stimulation. The whiter parts (arrows) in the maps b and c as compared to resting conditions show the contracting regions of the muscles in response to electrical stimulation.

Figure 3 : Typical PCr and contraction records carried out to calculate producer and consumer elasticities in a single animal. Muscle contraction and ${ }^{31} \mathrm{P}$ MRS spectra were measured simultaneously during modulations of the steady state. MR spectra were analyzed to assess steady values of energetic intermediates. The magnitude of the contraction signal was averaged over the corresponding period of time. Relative changes in contraction and [PCr] in response to modulations of the steady state provided all the required data for the calculation of the elasticities.

Figure 4 : Individual elasticity (A) and control (B) coefficients over the range from low to high muscle workload as a function of relative PCr level. Resting phosphocreatine concentration $[\mathrm{PCr}]_{\text {rest }}$ amounted to $27-28 \mathrm{mM}$. Over the range of selected electrical stimulations, the reference steady state $[\mathrm{PCr}]$ amounted to $19-20 \mathrm{mM}$ at low workloads, $16-17 \mathrm{mM}$ at moderate workloads and 11-12 mM at high workloads. Changes in elasticity and flux control coefficients as a function of muscle workload were approximated by mono-exponential functions. Exponential functions were drawn from least-square fits of elasticity data and then used to fit control coefficients using equation (2). Vertical dashed lines delimit the 3 sub-groups of data analysed as low, moderate and high workloads in table 1 and 2. 
Figure 1

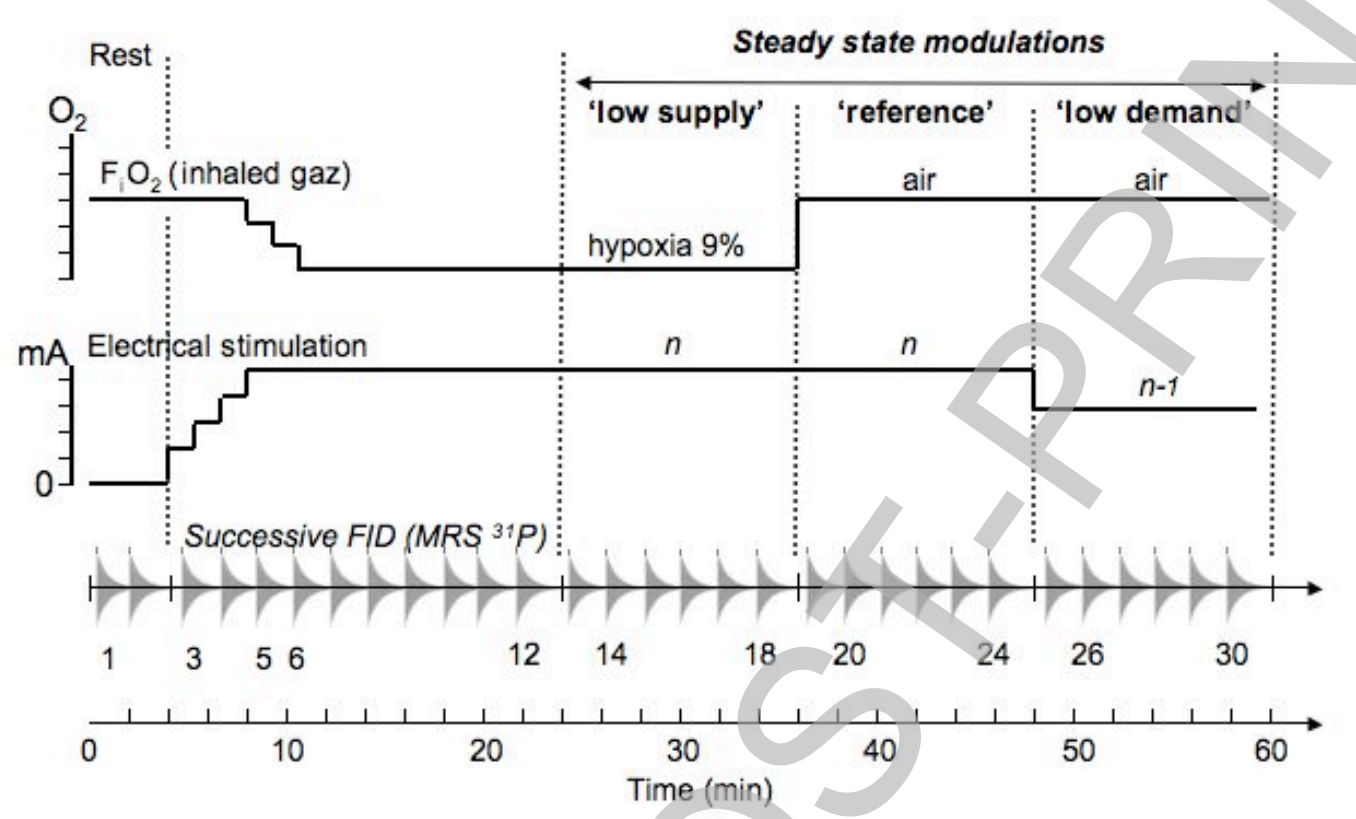

Licenced copy. Copying is not permitted, except with prior permission and as allowed by law. 
Figure 2

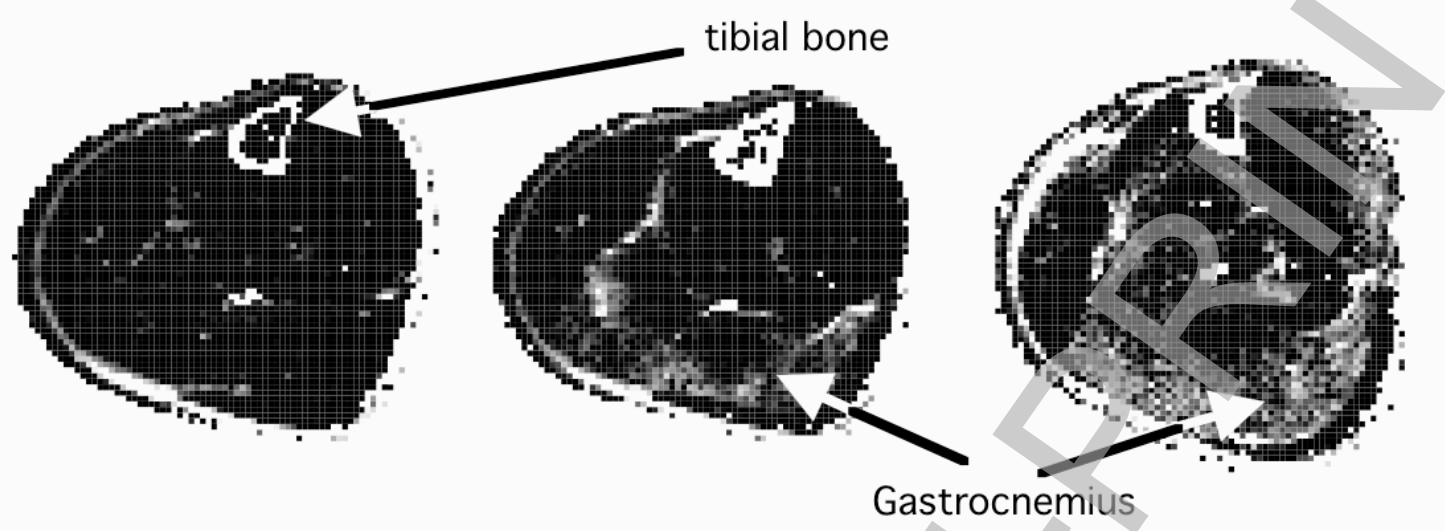


Figure 3

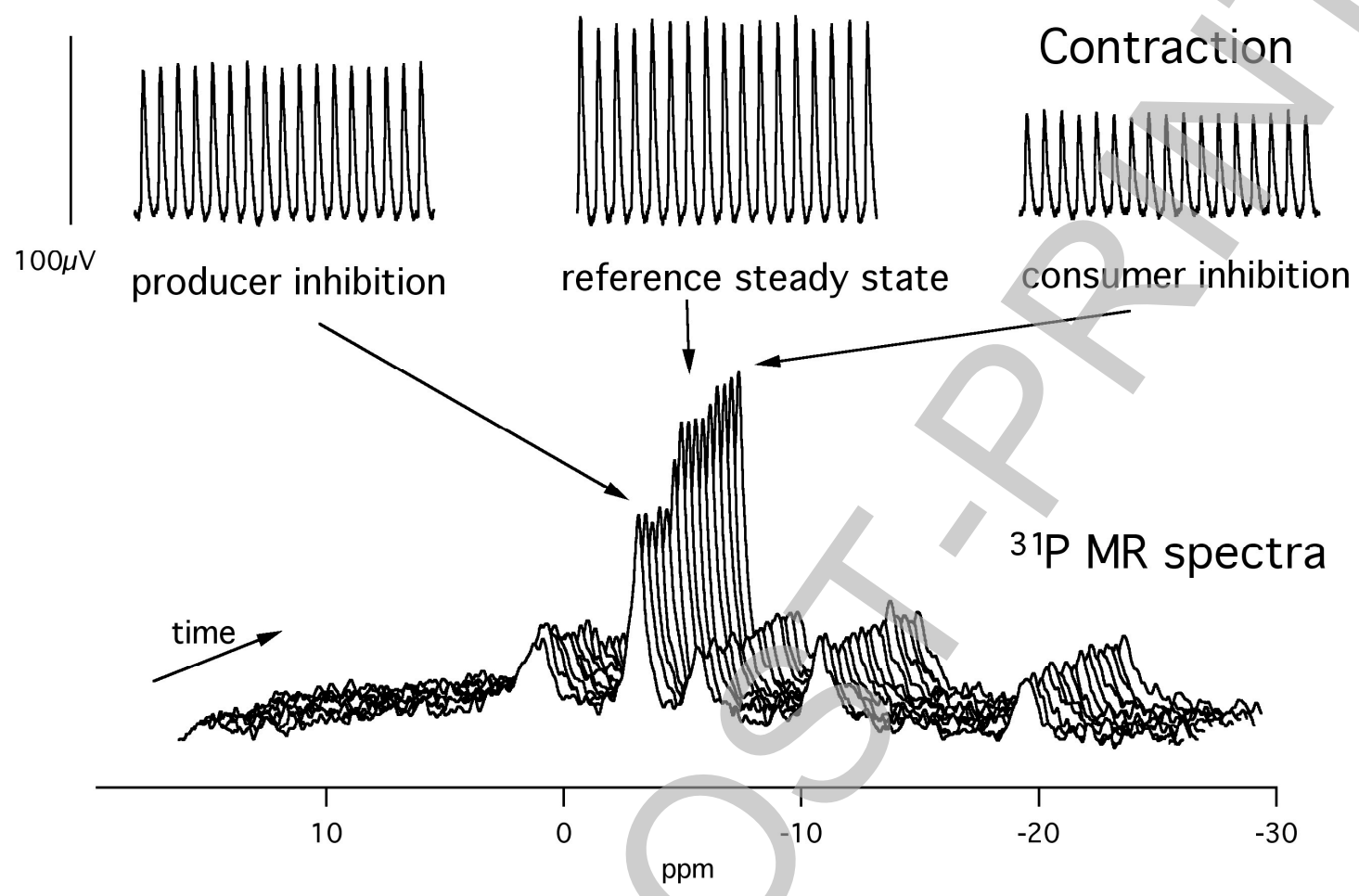


Figure 4
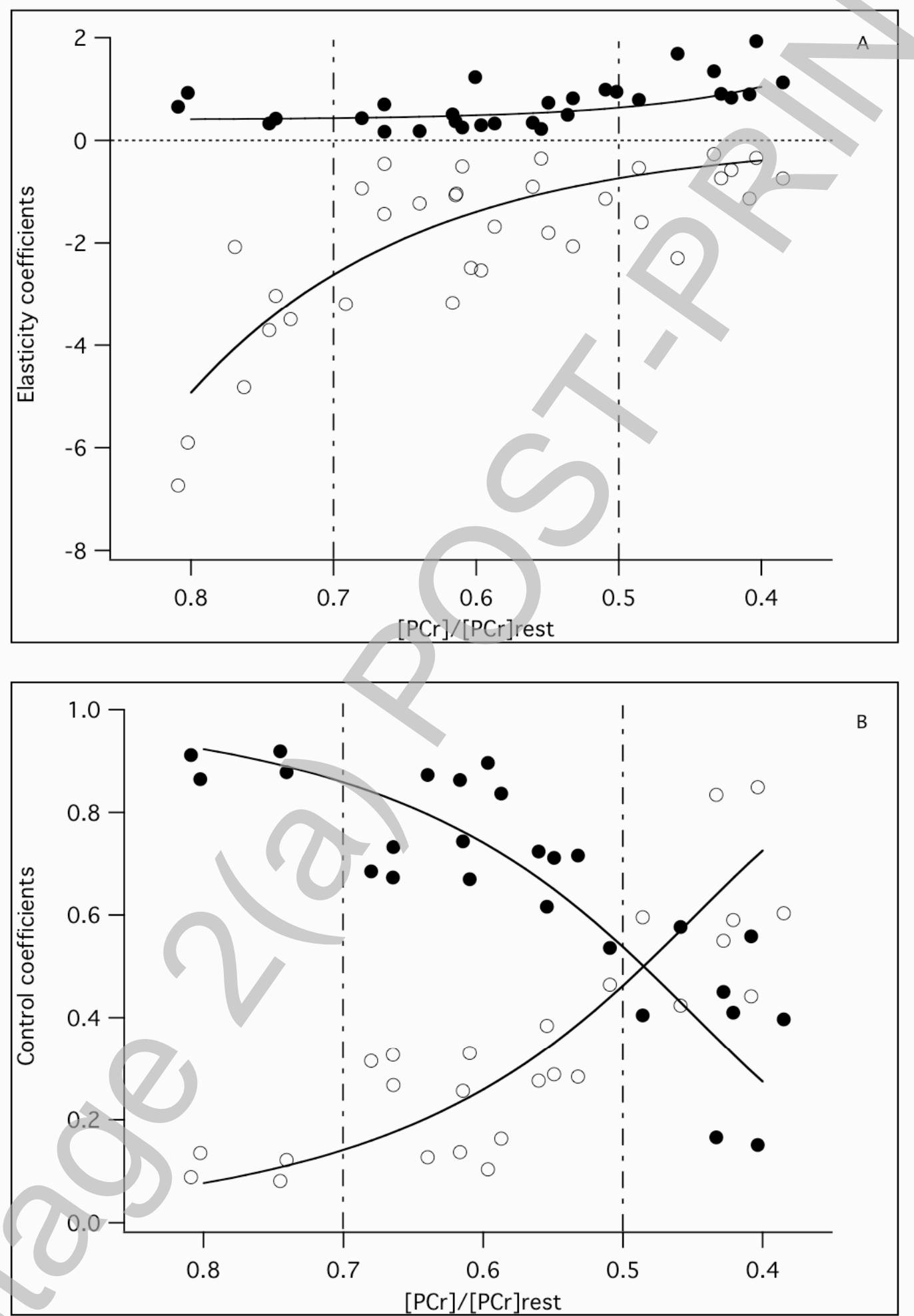

Licenced copy. Copying is not permitted, except with prior permission and as allowed by law. 